\title{
Juvenile Pemphigus Vulgaris as a Differential Diagnosis for Chronic Generalized Oral Ulceration in an Adolescent: A Case Report and a Review of Literature
}

\author{
${ }^{1}$ Kalani S Hettiarachchi, ${ }^{2}$ Ruwan D Jayasinghe, ${ }^{3}$ Chandira Gunasena, ${ }^{4}$ Primali Jayasooriya
}

\begin{abstract}
Pemphigus vulgaris (PV) is an autoimmune disorder involving the mucocutaneous tissues. Pathogenesis of this disease causes auto-antibodies against desmogleins in desmosomes which leads to intraepithelial blister formation. It is a rare but potentially life-threatening disease with a prevalence of 1 to 9 per $1 \times 10^{9}$. The disease has an equal sex predilection and commonly occurs in the 5th and 6th decade of life. Clinically, oral lesions are more evident than skin lesions. Diagnosis of the disease is by clinicopathological correlation with the definitive diagnosis requiring immunofluorescent investigations. The mainstay of treatment involves immunosuppression through the use of corticosteroids and other steroid-sparing agents like dapsone, azathioprine, and methotrexate. Presentation of this entity in an adolescent is a rare occurrence, and this may lead to misdiagnosis of the condition. Quality of life of these patients can be improved by controlling the disease through early diagnosis and necessary management. In this article, we report a case of a 15-year-old male patient diagnosed with Juvenile PV (JPV) and discuss the possibility of pemphigusvulgaris as a differential diagnosis for chronic generalized oral ulceration in an adolescent with a brief review of the literature.
\end{abstract}

Keywords: Juvenile, Oral ulceration, Pemphigus vulgaris.

How to cite this article: Hettiarachchi KS, Jayasinghe RD, Gunasena C, Jayasooriya P. Juvenile Pemphigus Vulgaris as a Differential Diagnosis for Chronic Generalized Oral Ulceration in an Adolescent: A Case Report and a Review of Literature. Int J Experiment Dent Sci 2018;7(2):143-146.

Source of support: Nil

Conflict of interest: None

\section{INTRODUCTION}

Pemphigus belongs to a group of rare autoimmune mucocutaneous diseases, which if left untreated can become life-threatening as a result of dehydration and superinfection. ${ }^{1}$ The family of pemphigus diseases consists of

\footnotetext{
${ }^{1}$ Senior Lecturer, ${ }^{2,4}$ Professor, ${ }^{3}$ Temporary Lecturer

${ }^{1-3}$ Department of Oral Medicine and Periodontology, Faculty of Dental Sciences, University of Peradeniya, Peradeniya, Sri Lanka ${ }^{4}$ Department of Oral Pathology, Faculty of Dental Sciences, University of Peradeniya, Peradeniya, Sri Lanka

Corresponding Author: Kalani S Hettiarachchi, Senior Lecturer, Department of Oral Medicine and Periodontology, Faculty of Dental Sciences, University of Peradeniya, Peradeniya, Sri Lanka, Phone: +94-081-2387451, e-mail: kalaniz2004@yahoo. com
}

the common entity of pemphigus vulgaris and its other variants such as pemphigus foliaceous, IgApemphigus, paraneoplastic pemphigus, and Hailey-Hailey disease. ${ }^{1}$ A pathognomonic feature of this disease entity is the appearance of fluid-filled blisters which rupture to give ulcers or erosions. The name pemphigus has been derived from the Greek terminology "pemphix" which means blister or bubble. ${ }^{1,2}$

Pemphigus vulgaris is the commonest disease of this entity ${ }^{1}$ with a prevalence of 1 to 9 per $1 \times 10^{9}$ per population. ${ }^{1,2}$ There seems to be racial predilection in this disease towards Ashkenazi Jews, individuals of Mediterranean and South Asian origins. ${ }^{1-3}$ Also, rare familial cases had been reported in literature. ${ }^{3}$ Genetic predilection is present in this disease which is related to Human Leukocyte antigen (HLA) class 2 phenotypes like HLA DR4, DRw14 and DQB. ${ }^{4}$ This phenotype seems to be present in other autoimmune diseases as well. ${ }^{4}$

The process of the pathogenesis in this condition occurs due to the production of auto-antibodies against desmogleins and desmocollins, an integral part of epidermal cell-cell adhesion which causes acantholysis leading to intraepithelial blister formation. ${ }^{4,5}$ Exact mechanism causing this acantholysis is not elucidated, but many theories have been put forward. ${ }^{1,4}$

Pemphigus vulgaris involves both the oral cavity and the skin. ${ }^{1,2}$ In $60 \%$ of the cases, the oral lesions are followed by skin lesions. ${ }^{1,2,5}$ Posterior buccal mucosa, palate, and gingiva ${ }^{1}$ are the areas commonly involved in the oral cavity and when the gingival involvement is seen it is named as desquamative gingivitis. ${ }^{1,2}$ However, the involvement of pharynx and larynx has also been reported. ${ }^{1,2}$ Skin involvement of this disease mainly manifests in the head, scalp, neck and then spreads to the trunk and flexor areas of the limbs. ${ }^{1,3}$

There are no specified criteria for the diagnosis of this condition; diagnosis is mainly by clinical, immunofluorescence assays and histopathological correlation. The mainstay of treatment involves immunosuppression through the use of corticosteroids used either topically or systemically and other steroid-sparing agents like dapsone, azathioprine, and methotrexate. ${ }^{1,2,5}$

Presentation of pemphigus vulgaris in an adolescent is extremely rare. ${ }^{5,6}$ Only a few cases have been reported 
Table 1: Summary of literature pertaining to adult pemphigus vulgaris and juvenile pemphigus vulgaris

\begin{tabular}{llll}
\hline & Adult pemphigus & Juvenile pemphigus & Present case \\
\hline Age (years) & $50-60$ & $3.5-18$ & 15 \\
Gender & Equal predilection & Slight female predilection & Male \\
Racial predominance & Ashkenazi Jews, individuals of & No adequate information & South Asian \\
& Mediterranean and South Asian origins. & & Same but absent in the \\
Oral Lesions & $\begin{array}{l}\text { Posterior buccal mucosa, palate, } \\
\text { gingiva and anywhere in the mouth }\end{array}$ & Same & gingiva \\
Skin Lesions & Appears after oral lesions & Appears after oral lesions & Appeared before skin lesions \\
Histopathology & Intraepithelial blistering & same & same \\
Immunofluorescence & Positive & Positive & Positive \\
Treatment & Steroids and steroid sparing agents & Steroids and steroid sparing agents Steroid only \\
\hline
\end{tabular}

in the literature (Table 1$).{ }^{5}$ Therefore, misdiagnosis of this condition in a patient presenting with oral ulcerations is extremely high, and this may lead to a delay in diagnosis and proper management. ${ }^{5-7}$ Early diagnosis is paramount important for proper intervention as that will help to control this condition and thereby improve the quality of life in young patients. ${ }^{5}$ Here we the present case of juvenile pemphigusvulgaris to show the importance of considering pemphigus vulgaris as a differential diagnosis for chronic oral ulceration.

\section{CASE HISTORY}

A 15-year-old male patient was referred to the Oral Medicine clinic, University Dental Hospital Peradeniya, Sri Lanka by a medical officer for the management of recurrent oral ulcerations. He has been suffering from recurrent ulcerations for 2 years without an ulcer-free period despite the treatments received. His past medical history and family history was of no significance. On examination, the patient looked depressed, and there was evidence of healed lesions as scars on the face, scalp, and back. Intraorally there were multiple ulcers present on the bilateral buccal mucosa and the tongue (Fig. 1). The margins of the ulcers were erythematous, and the base was whitish, and the patient was also noted to have poor oral hygiene.

Different diagnosis of vesiculobullous disorder was considered, and a full blood count (FBC) and anincisional biopsy were performedon the same day with the informed consent from the child's mother. Both fixedand freshsamples were sent for histopathology and immunofluorescence studies, and the FBC findings were not significant.

Incisional biopsy reported intraepithelial blistering with acanthosis in the parakeratinized stratified squamous epithelium. However, the results of direct immunofluorescence (DIF) for IgG, IgA, and C3 antibodies were negative, and these findings were suggestive of Benign Familial pemphigus (Hailey-Hailey disease). As the family history was negative and histopathological features were confirmatory, a repeat biopsy for immuno fluorescence was considered to confirm the diagnosis.
The repeat DIF revealed light intercellular IgG positivity. Therefore, based on clinicopathological correlation, a definitive diagnosis of juvenile pemphigus was confirmed, and the patient was prescribed with a tapering dose of Prednisolone $10 \mathrm{mg}$ for 1 week together with a mouth wash. On this review appointment, the severity of the ulcers was reduced, and the patient felt better, therefore, the same drug regime was prescribed, and the patient was referred to a dermatologist for further management.

\section{DISCUSSION}

Pemphigus vulgaris (PV), usually considered to be a disease of middle age, occurs commonly in the 5th and 6th decades of life and only a handful of cases of Juvenile pemphigusvulgaris are reported in the literature. Pemphigusvulgaris affecting individuals below 20 years of age has been described using various terms such as 'juvenile PV', 'adolescent PV', 'childhood PV' and 'pediatric PV'. ${ }^{5}$ Since such terminology confuses, Gorsky et al. suggested, using the terms 'childhood PV' and 'adolescent $\mathrm{PV}^{\prime}$ to describe the condition in individuals under 12 years of age and in those aged between 12 and 18, respectively. ${ }^{8}$

Even though, some authors report slight female predilection in cases of juvenile pemphigus ${ }^{5}$ generally this disease has equal sex predilection. ${ }^{1,2}$ Usually in pemphigus, oral lesions occur before skin lesions. ${ }^{7-9}$ In contrast, our patient presented with skin lesions which were preceded by oral lesions.

Further, the commonest sites of involvement are the bilateral buccal mucosae, gingiva, and palate. ${ }^{8}$ However, this case presented with the involvement of the buccal mucosa and the tongue with no desquamative gingivitis. ${ }^{1,2}$ The involvement of the skin was inconsistent with the literature.

When a patient presents with generalized chronic ulceration in the oral cavity, the differentials to be considered are pemphigus vulgaris, erythema multiforme, acute herpetic gingivostomatitis, bullous impetigo, linear IgA disease, epidermolysis bullosa, cicatricial pemphigoid, bullous pemphigoid and paraneoplastic 

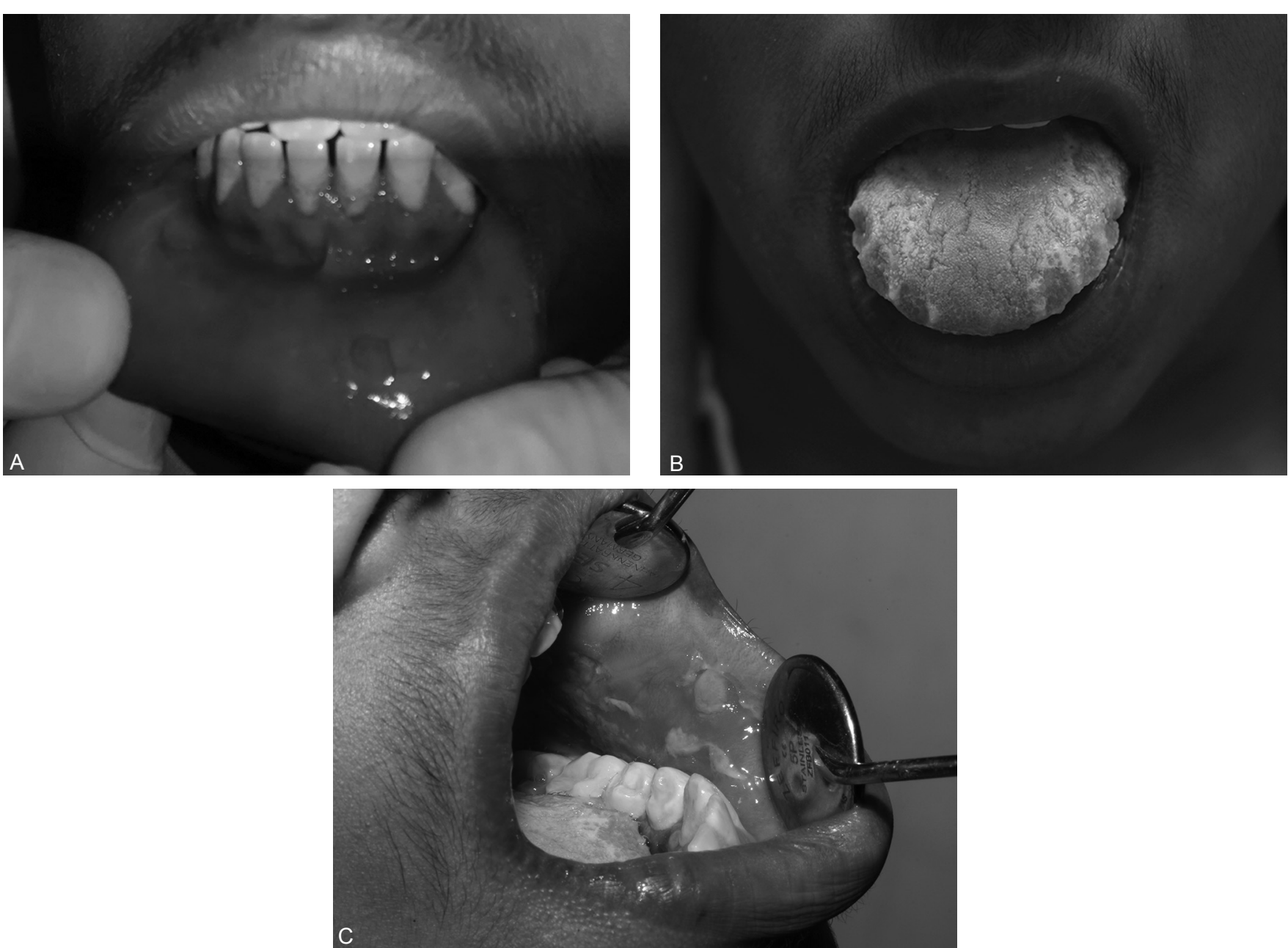

Figs $1 \mathrm{~A}$ to $\mathrm{C}$ : (A) Ulcers on the labial muocsa; $(\mathrm{B})$ Indentations on the tounge; (C) Ulcers on the left buccal mucosae

pemphigus. ${ }^{78}$ However, especially in an adolescent, the possibility of pemphigus vulgaris, erythema multiforme, acute herpetic gingivostomatitis, and aphthous ulcers need to be considered. ${ }^{5}$ In this case presented here due to lack of ulcer-free period the possibility of aphthous ulcers becomes less likely and further, as there are neither clinical features suggestive of infection nor immunodeficiency status acute herpatic gingivostomatitis can also be excluded. ${ }^{5}$ The possibility of epidermolysisbullosa, cicatricial pemphigoid, bullous pemphigoid, and paraneoplastic pemphigus conditions was not taken into consideration as the clinical features were not very suggestive. Therefore, the clinical features were more in favor of a vesiculobullous disease.

Desmogleins are cadherin-like proteins which are components of cell junctions constitute an integral part of the desmosomes. ${ }^{8}$ Cell junctions adhere cells together and maintain the integrity of mucous membranes and skin. In pemphigus, there is autoantibody production against desmoglenins, and these antibodies cause the detachment of keratinocytes from each other causing the formation of intraepithelial blisters which is a histopathological feature seen in this group of diseases. ${ }^{1,4}$ Out of the two types of desmogleins, antibodies against desmoglenin ${ }^{3}$ and anti- bodies against desmoglenin ${ }^{1}$ is seen predominantly in a patient with oral lesions, and skin lesions respectively ${ }^{1,4}$ is predominantly seen in patients with skin lesions. ${ }^{1,4}$

Even though the pathogenesis of this disease is known as the reason for the breakdown of desmosomes after binding with the autoantibodies remains a gray area. ${ }^{4}$ Many theories like Steric hindrance theory, desmoglein compensation theory, multiple hits-hypothesis, and antibody-induced apoptosis theory ${ }^{4}$ are reported in the literature with futile results. ${ }^{4}$ Interestingly the theory on "Apoptosis" which relates the suprabasal acantholytic and cell death pathways to basal-cell shrinkage seems promising. ${ }^{4}$

Usually, the histopathological diagnosis is not confirmatory in the diagnosis of pemphigus vulgaris. Immunofluorescence is performed on a fresh perilesional biopsy to identify antibodies to $\operatorname{IgG}, \operatorname{IgA}$, and C $3 .{ }^{1}$ In this patient, the initial biopsy was reported to be negative to the above-mentioned antibodies directing the diagnosis of Hailey-Hailey disease (HHD), or familial benign chronic pemphigus which is anautosomal dominant blistering skin disease. ${ }^{9,10}$ However, in contrast, the pathogenesis of HHD involves mutations resulting in inactivation of an allele of ATP2C1 which encodes 
the human secretory pathway $\mathrm{Ca} 2+/ \mathrm{Mn} 2+$-ATPase protein 1 (SPCA1) that is found in the Golgi apparatus. SPCA1 protein is located in keratinocytes which are involved the differentiation, maturation, and production of keratinocytes, therefore, any abnormality will cause loss of cell adhesion and leads to intraepithelial blistering. ${ }^{9}$ As a result of positive histopathology with a negative immune fluorescence the possibility of HHD was considered. However, with a negative family history and no suggestive clinical features such as blistering in the inguinal and axial regions and lack of white longitudinal lines in the fingers this diagnosis was reconsidered and a repeat biopsy for immunofluorescence was soughed. With a weak positivity, the diagnosis of juvenilepemphigusvulgaris was confirmed. In the literature, Arbache et al. have reported DIF has a high sensitivity in diagnosing pemphigus vulgaris. ${ }^{11}$ The reasons for a negative DIF may be due to the administration of steroids prior to obtaining tissue for biopsy $y^{2,11}$ or a technical glitch as in this case.

The treatment of pemphigus vulgaris is based on immune suppression which is achieved by administration of a local or a systemic steroid or a steroid-sparing drug or a combination and this clinical decision mainly depends on the severity of the disease. ${ }^{1,2,5}$ The novel trends of treatment include a combination of steroids with steroid-sparing drugs like Dapsone, azathioprine, methotrexate, cyclophosphamide, gold, and cyclosporine which serves as adjuvants. ${ }^{2}$ According to the literature even though systemic immunosuppression is still largely used, recently there have been attempts at more specific modulation of the autoimmune response which requires autoreactive helper T-cells that regulate immunoglobulin isotype switching. ${ }^{2}$ Further, with proper management of the condition with the use of steroids has reduced the mortality rates of up to $10 \%$.

Usually, for systemic steroids, the oral route is preferred as it noninvasive,easy, with no pain involved. Intravenous route of administration is also used withgood results. ${ }^{2}$ The drug of choice is prednisolone. An initial dose of 100-150 mg prednisolone alone or with azathioprine (100-150 mg) and a maintenance dose of 5-20 mg daily for varying periods has been recommended for adult patients. ${ }^{5}$ For juvenile cases, the dose should be tailored according to the patient's age, weight, and severity of tehcondition. ${ }^{5}$ Ariyawardena et al. reports the use of combinations of drugs like Azathioprine and intravenous immunoglobin therapy with effective results in the management of this condition. ${ }^{5}$ Literature reports minimal chances of remissions and even occurred lasts only for a short period ${ }^{2}$. However, there is a lack of evidence with regard to the prognosis using different drug regimes in juvenile pemphigus. ${ }^{2,5}$
It is mentioned in the literature that early diagnosis of juvenile pemphigus will lead to good prognosis. ${ }^{2,5,6}$ However, in this case, there was a delayed presentation due to misdiagnosis and improper treatment. ${ }^{5-7}$ This was identified as a problem in the case reports reported by Srivastava et al., Dyer et al., and the majority of case reports summarized by Ariyawardena et al..$^{5-7}$ This results due to lack of awareness of the clinicians' when considering differential diagnosis in patients presented with chronic generalized ulcerations especially in adolescents.

\section{CONCLUSION}

Here we present a rare case of pemphigus with oral involvement in an adolescent, which was diagnosed by clinicopathological correlation. In the end, it is important to consider juvenile pemphigus as a differential diagnosis in the presentation of chronic oral ulceration and thereby initiate the treatment at early stages of the disease for better prognosis with improved quality of life.

\section{REFERENCES}

1. Warnakulasuriya S, Tilakaratne WM. Oral Medicine and Pathology: A Guide to Diagnosis and Management. Jaypee brothers. 2014 1/e, 9789350252215

2. Scully C, Challacombe SJ. Pemphigus Vulgaris: update on etiopathogeneis, oral manifestations and management. Crit Rev Oral Biol Med 2002;13(5):397-408

3. Gregoriou S, Efthymiou O, Stefanaki C, et al. Management of pemphigus vulgaris: challenges and solutions. Review. Clinical, Cosmetic and Investigational Dermatology, Dove Press 2015;8:521-527.

4. Madala J, Bashamalla R, Kumar MP. Current concepts of pemphigus with a deep insight into its molecular aspects. J Oral Maxillofac Pathol 2017;21:260-263.

5. Ariyawardena A, Tilakaratne WM, Dissanayaka M, et al. Oral pemphigus vulgaris in children and adolescents: a review of the literature and a case report. International Journal of Paediatric Dentistry 2005;15:287-293.

6. Jacyk WK, Dyer RB. Juvenile pemphigus vulgaris. A case report. South African Medical Journal 1987;71:325.

7. Srivastava A, Poornima G, Balaji P, et al. Juvenile Pemphigus Vulgaris. Int J Prev Clin Dent Res 2017;4(1):1-4.

8. Gorsky M, Raviv M, Raviv E. Pemphigus vulgaris in adolescence. A case presentation and review of literature. Oral Surgery, Oral Medicine and Oral Pathology 1994;77: 620-622.

9. Cheng TS. A review on Hailey-Hailey disease. Review Article. Hong Kong J. Dermatol. Venereol. 2007;15:10-16.

10. Tcherne VG, Cardoso JC. Familial benign chronic pemphigus (Hailey-Hailey Disease): use of topical immunomodulators as a modern treatment option. Rev Med Chile 2011;139: 633-637.

11. Arbache ST, Delgado l, Aoki V, et al. Immunofluorescence testing in the diagnosis of autoimmune blistering diseases: overview of 10-year experience. Investigation. An Bras Dermatol. 2014;89(6):885-889. 